\title{
Implementação de um Sistema de Monitoramento de Utilização de Máscaras em Faces Utilizando Algoritmo de Inteligência Artificial
}

\author{
João Pedro Lourenço Monteiro ${ }^{1}$, Geraldo Castro Carvalho Junior ${ }^{1}$, Yonara Costa \\ Magalhães ${ }^{1}$, Will Ribamar Mendes Almeida ${ }^{1}$. \\ ${ }^{1}$ NuSTI - Núcleo de Pesquisa em Sistemas e Tecnologia da Informação \\ Curso de Engenharia de Computação \\ Universidade CEUMA - São Luís, MA - Brazil \\ joaopedromonteiro2014@gmail.com,juniorcastrz@gmail.com \\ yonara.magalhaes@ceuma.br, will75@gmail.com
}

\begin{abstract}
Artificial Intelligence has gained strength in the area of computer vision for face recognition. In the context of COVID-19, the application of facial monitoring was extended to the identification of faces using protective masks. It is proposed to build a solution using Machine Learning techniques to detect protective masks and, later, generate data that support public policies regarding the mandatory use of these masks. As preliminary results, the classification model was developed, from an image bank, of this monitoring whose evaluation metrics were satisfactory.
\end{abstract}

Resumo. A Inteligência Artificial tem ganhado força na área de visão computacional para reconhecimento de rostos. No contexto da COVID-19, a aplicação de monitoramento facial foi estendida para a identificação de rostos que utilizam máscaras de proteção. Propõe-se construir uma solução utilizando técnicas de Machine Learning para detecção de máscaras de proteção e, posteriormente, gerar dados que subsidiem as políticas públicas quanto à obrigatoriedade do uso destas máscaras. Como resultados preliminares, foi desenvolvido o modelo de classificação, a partir de um banco de imagens, deste monitoramento cujas métricas de avaliação foram satisfatórias.

\section{Introdução}

Quando há um local com alto risco de contaminação biológica é comum, e faz parte dos protocolos sanitários, usar equipamentos para evitá-la. Este cuidado existe nas profissões que estão sujeitas a este tipo de risco. Bem como, também são adotadas no enfrentamento à disseminação de doenças, caso fundamental no combate à pandemia da COVID-19. Um desses métodos refere-se ao uso de máscara, já comprovado sua eficácia na retenção de gotículas liberadas ao falar, tossir etc, que é uma das principais formas de contaminação direta por vírus respiratórios (Van Der Sande e col, 2008). Apesar da ciência já comprovar a eficácia do uso da máscara, é comum observar pessoas utilizando a máscara de forma incorreta ou simplesmente não usando-a. O que contribuiu para a aplicação de uma lei sobre o uso obrigatório da máscara (Lei $\mathrm{N}^{\circ}$ 14.019, de 2 de julho de 2020) apesar da sua vigência, a lei por si não garante o cumprimento proposto em sua plenitude, pois não é possível apurar com precisão se a obrigatoriedade está sendo de fato cumpridas. 
Partindo do contexto mundial atual, o presente trabalho visa desenvolver uma ferramenta computacional capaz de ajudar no combate a disseminação doença da COVID-19, auxiliando as autoridades interessadas, dispondo de dados mais precisos quanto ao cumprimento do uso da máscara, bem como aumentar a segurança dos funcionários, do setor privado e público, que são designados a fazer o controle da entrada do estabelecimento, evitando o contato desnecessário com outras pessoas. Esta ferramenta partiria de tecnologias já existentes, disponíveis no campo da visão computacional

\section{Fundamentação Teórica}

Segundo Resende e Pereira (2015, p. 4) é fato que os humanos possuem uma visão privilegiada, permitindo-lhes distinguir com facilidade, características tais como, formas, uma vasta gama de cores, emoções, já o ambiente computacional, como um todo, não consegue reproduzir esse feito com tanta facilidade.

\subsection{COVID-19}

Segundo o Ministério da Saúde, a Covid-19 é uma infecção respiratória aguda causada pelo coronavírus SARS-CoV-2, com muitos casos graves e alta taxa de transmissibilidade, culminou em uma pandemia global. Segundo Garcia (2020), pelo fato de ser uma doença respiratória, a contaminação é muito fácil de ocorrer, sendo necessário apenas um espirro ou levar as mãos contaminadas aos olhos, boca, etc. Em situações como essa, as autoridades sanitárias recomendam o uso de medidas chamadas de Intervenção Não Farmacológica (INF).Para tanto, a partir de abril de 2020, o Ministério da Saúde também passou a orientar a população para o uso de máscaras faciais de pano, cirúrgica ou padrão N95, para atuarem como barreira à propagação da SARS-CoV-2.

\subsection{Detecção de faces}

A área de estudo que tenta dar à máquina a capacidade de "ver" é a da visão computacional. Nesta área, é possível detectar faces e, até mesmo, fazer o reconhecimento de pessoas. Uma das técnicas utilizadas para o reconhecimento é o uso de Machine Learning, que também será utilizado no desenvolvimento do algoritmo. Para Zhao et al (2003) detectar faces é a capacidade do algoritmo localizar e destacar, de alguma forma, um rosto de uma pessoa em uma imagem, seja ela vídeo ou foto.

Para alcançar o objetivo de identificar se a pessoa está ou não usando máscara, o algoritmo irá detectar, no vídeo, as faces das pessoas para enfim aplicar sobre elas o classificador de máscaras, a detecção das faces é feita usando dois arquivos deploy.prototxt que define a arquitetura do modelo e o .caffemodel que se trata do modelo em questão, a qual é uma deep neural network (DNN) ou Rede Neural Profunda disponível na biblioteca OpenCV a escolha se dá pelo simples fato de ser mais eficiente que os outros modelos em relação a performance, foi foi treinada com milhões de imagens) trazendo segurança na detecção de faces (Documentação OpenCV, 2019). 


\subsection{Métricas de Avaliação}

Segundo Mario Filho (2021), no processo de criação de um modelo de aprendizado de máquina é necessário medir sua qualidade de acordo com os objetivos da tarefa. Existem algumas funções matemáticas que ajudam a avaliar o erro e a correção do modelo. Essas medições serão chamadas de Métricas de Avaliação e hiperparâmetros. A seguir, apresenta-se uma breve explicação sobre estas métricas de avaliação:

a) Precision: Dentre todas as classificações atribuídas como positivo que o modelo fez, quantas estão corretas.

b) Recall: Razão dados classificados como positivos quantos são verdadeiramente positivos.

c) F1-score: Pode ser entendida como uma união das duas anteriores, ele reflete os valores da precisão e recall pois se algum deles for baixo o resultado irá refletir em um F1-score baixo.

d) Acurácia: Esta mede a quantidade de acertos sobre o todo. Ou seja, se refere ao percentual de classificações feitas corretamente, como ela representa o todo temos apenas um valor.

e) Taxa de aprendizagem: Definida com valor de 0,0001.

f) Época: Com valor de 20 .

g) Tamanho do lote: 32 .

\section{Metodologia}

Este trabalho se caracteriza por ser uma pesquisa aplicada que pretende desenvolver solução que utiliza IA para reconhecer se as faces humanas estão ou não utilizando máscaras de modo a quantificar essas pessoas, em um determinado espaço físico ou local e, desta forma, realizar ações de monitoramento ou de fiscalização mais eficientes, principalmente em locais com alto fluxo de pessoas. Tais dados comporão um banco de dados, organizados por dia e horário (bem como demais parâmetros julgados essenciais), para composição de informações estatísticas e precisas sobre a utilização de máscara e, ao mesmo tempo, gerar alertas sobre as condições como produto deste monitoramento.

\subsection{Percurso metodológico}

O trabalho está estruturado nas seguintes etapas:

A. Foi realizada pesquisas bibliográficas sobre Covid19, Inteligência Artificial, reconhecimento de face, aprendizagem de máquina, métricas de avaliação e pesquisas sobre banco de imagens e softwares adequados para utilização da construção da solução proposta;

B. O desenvolvimento do modelo de classificação, foi feito a partir da aquisição de um banco compartilhado de imagens, da Prajna Bhandary, e prosseguiu-se com a adoção de uma rede neural pré treinada, MobileNetV2, para detectar as faces para então realizar a detecção de máscaras faciais, 
com o algoritmo sendo capaz de classificar faces com e sem máscaras. $\mathrm{Na}$ seção 3.2, deste, são detalhadas as ferramentas, algoritmos e plataformas utilizadas;

C. Modelagem da solução: em relação ao banco de dados da aplicação, a interface da solução, a delimitação dos parâmetros de coletas e os tipos de alertas de monitoramento, estas atividades ainda estão em processo de desenvolvimento. Pois a intenção, é que a partir das imagens capturadas durante a classificação seja possível armazenar em um banco de dados, para segmentação, identificação, classificação e geração de dados;

D. Estão sendo realizados testes e simulação, ao longo de todo o processo de desenvolvimento, em um ambiente controlado, verificando o treinamento da rede neural, a captura de imagens, a detecção de máscaras de proteção e realizando os ajustes necessários no protótipo. Isto permitirá sua validação em um ambiente real.

E. Foi realizada uma sessão de detecção com objetivo de mensurar o quão bem estão sendo precisas as capturas do algoritmo, quanto na detecção de rostos quanto na classificação deles, o local escolhido foi a porta de entrada principal do Shopping Rio Anil (São Luis-MA), no período noturno de pico. Para isso foi usado um notebook, com as configurações descritas no $3^{\circ}$ parágrafo deste tópico, para executar o algoritmo, juntamente com uma câmera de um celular vinculada ao notebook por meio do aplicativo DroidCam e uma estrutura fixada ao solo para apoiar todo o conjunto.

O código utilizado neste trabalho tem como foco a classificação em tempo real de rostos de pessoas utilizando máscara, seguida de uma explicação detalhada das principais métricas, bibliotecas e técnicas adotadas no desenvolvimento do algoritmo. O desenvolvimento do presente trabalho dá-se pela utilização de uma câmera, de preferência externa ao computador, que servirá para capturar as fases, e de um computador que ficará executando o algoritmo e armazenando as faces que ele conseguir classificar para depois serem apresentadas diante de uma apuração a fim de estipular as porcentagens de precisão e acurácia alcançadas.

Este trabalho foi desenvolvido com um processador Intel(R) Core(TM) i5-8250U CPU @ $1.60 \mathrm{GHz}$ e uma placa de vídeo dedicada NVIDIA(R)GeForce(R)MX150 de 2GB. Para o desenvolvimento foi utilizado Python e Open $C V$, que juntos trazem a simplicidade da linguagem com o estado da arte em visão computacional e Deep Learning, elaborado usando a IDE PyCharm.

O conjunto de dados utilizados foi compartilhado pela Prajna Bhandary em seu GitHub, são 1.376 imagens divididas em 690 imagens de faces com máscara e 686 sem máscaras. Prajna criou esse conjunto de dados usando OpenCv para localizar a face na imagem e extrair dela a região de interesse chamada de ROI e então aplicar na face um detector de pontos faciais (landmarks) por meio da biblioteca Dlib, permitindo mapear a estrutura facial do rosto localizando nela o nariz, boca, olhos, rosto. Desta forma, aplicar a imagem de uma máscara (com fundo transparente) nas landmarks corretas, com isso ela criou um conjunto artificial de dados, porém utilizáveis em mundo real (Figura 1). 
Figura 1. Imagens usadas no treinamento.
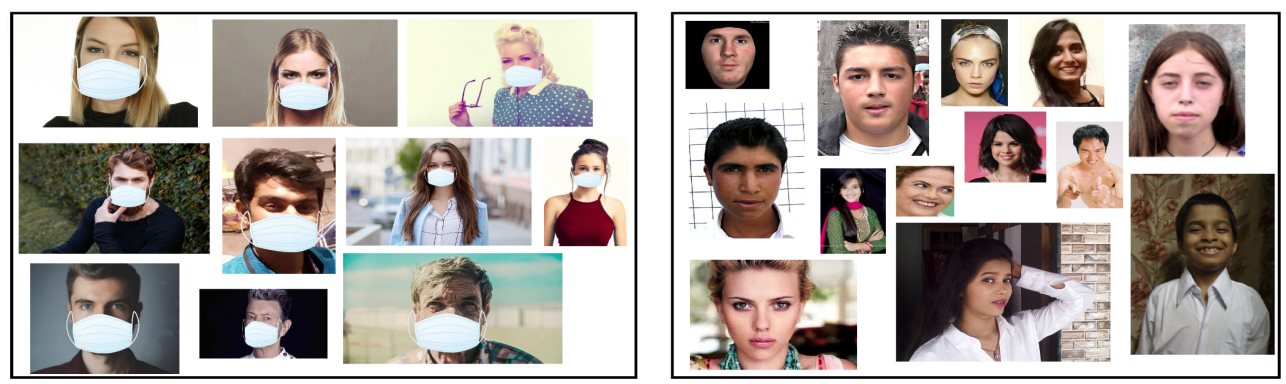

Fonte: Banco de imagens usada pelo autor.

A aplicação das máscaras virtuais é feita através de aplicação de pontos faciais para localizar olhos, boca, nariz e ouvidos. Antes de mais nada é preciso localizar a face de uma pessoa para então aplicar os pontos faciais no rosto dela. Para tanto foi usada a biblioteca Dlib, ela foi desenvolvida para trabalhar com visão computacional, aprendizagem de máquina e reconhecimento de faces, através dela é possível aplicar a detecção facial em cima de uma face permitindo encontrar nela vários pontos faciais, chamados de landmarks. $\mathrm{O}$ algoritmo está dividido em duas classes principais: video detector de mascaras.py responsável por iniciar a câmera definida e capturar as faces a fim de classificá-las e treino_detector de mascara.py onde é feito treino do modelo de classificação. Os dados do dataset foram divididos em dois conjuntos de treino e teste, $80 \%$ dos dados para treino e $20 \%$ para teste, os dados de treino foram utilizados para criar um modelo de classificação de faces com máscara ou não, para isso foi usada a rede neural MobiNetV2.

A MobileNetV2 utiliza o dataset ImageNet. Esse dataset faz parte de um projeto que visa fornecer um grande banco de imagens para fins de pesquisa, e contém mais de 14 milhões de imagens que pertencem a mais de 20.000 classes. Com a MobileNetV2 é possível treinar redes neurais profundas e complexas, que geram excelentes resultados. Se faz interessante usar a MobileNetV2 por conta das camadas de convolução em profundidade para construir redes neurais profundas o que reduz a complexidade do modelo, dando utilidade para sistemas embarcados com menor poder de processamento, porém sendo tão eficiente quanto outros modelos na tarefa de classificar cada face em uma imagem (ANDREW G. HOWARD e col. 2017).

\subsection{Ferramentas}

A linguagem Python foi escolhida justamente por oferecer uma gama grande e poderosa de programação em visão computacional e machine learning oferecida a partir das bibliotecas atreladas a ela, algumas delas foram usadas na concepção do código.

Utilizou-se o Tensor Flow, que é uma biblioteca de código aberto criada pelo Google em 2015 especificamente pela equipe Google Brain, com ela será possível 
trabalhar com redes neurais e treinar modelos de Deep learning de forma mais prática e fácil, já que ela possui toda estrutura pronta para o trabalho. (Didática Tech, 2020). Para auxiliar no desenvolvimento com Tensor Flow foi utilizado no projeto a API Keras, ela é executada em cima da plataforma Tensor Flow. O Keras foi criado para ser, escalável, fácil de estender e trabalhar usando o Python. "A API foi projetada para seres humanos, não para máquinas e segue as melhores práticas para reduzir a carga cognitiva" (Documentação Keras, 2020).

Em conjunto com o Tensor Flow, foi usada a biblioteca OpenCv, que é um software de código aberto voltado para visão computacional e para aprendizado de máquina. O Open $C v$ dispõe de algoritmos para detecção de faces, que foi selecionada e utilizada no projeto para identificar as faces capturadas pela câmera.

\section{Resultados e discussões}

Até o momento foram realizadas as etapas de pesquisa bibliográfica e desenvolvimento do algoritmo utilizado um banco de imagens compartilhado como treinamento para o modelo de classificação de faces. Obtendo os resultados descritos a seguir.

Os resultados preliminares, alcançados na etapa de teste, foram obtidos usando o método classification_report(), do qual obteve-se os resultados referentes às métricas de:

a) Precisão: Para a classe com máscara se obteve uma precisão de $99 \%$ e 94\% para sem máscara.

b) Recall: Para a classe com máscara se obteve um recall de $91 \%$ e $99 \%$ para sem máscara.

c) F1-score: Para a classe com máscara se obteve um f1-score de 95\% e 97\% para sem máscara.

d) Acurácia: Sendo uma porcentagem de $96 \%$ de acurácia.

permitindo avaliar antes de realizar as detecções se o modelo possui uma boa capacidade de generalização. $O$ fato da taxa de aprendizagem ser bem pequena acarretou em um tempo de treino bem demorado, em virtude das configurações da máquina, porém foi alcançada valores de perda (train_loss e val_loss) muito baixo, tanto na etapa de treino quanto de teste (figura 4), o que reflete também em uma boa acurácia (train_acc e val_acc) ao apresentar imagens novas.

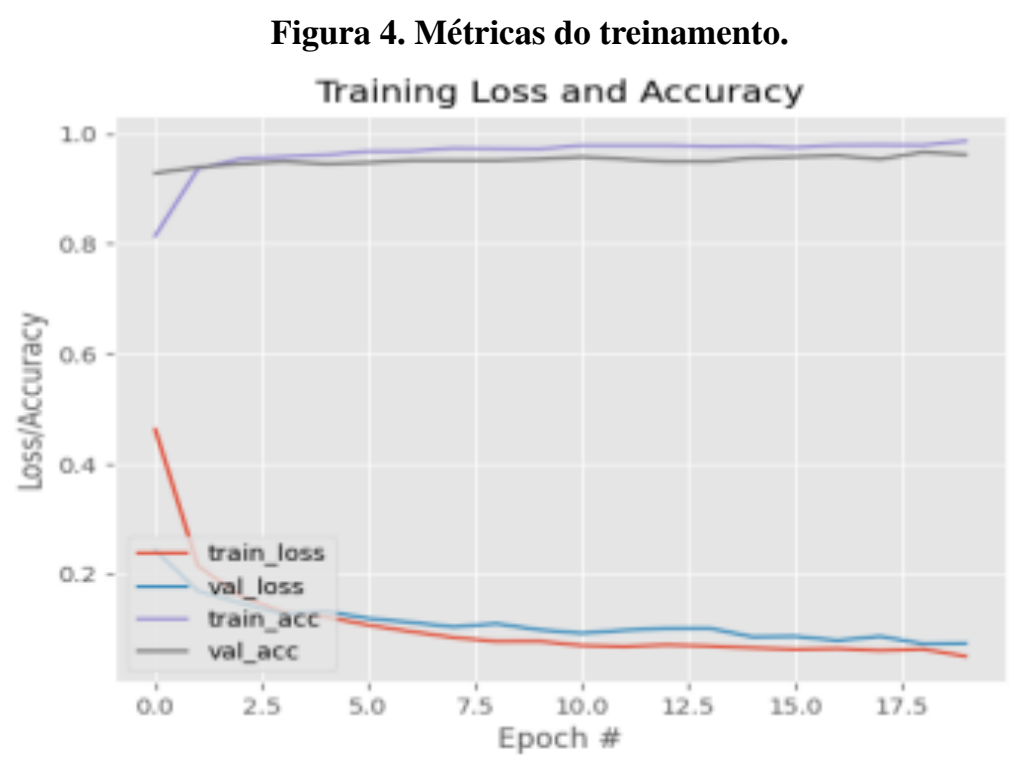


Os resultados, até o momento, permitiram explorar a implementação de teste em um ambiente com alto fluxo de pessoas, possibilitando observar o comportamento do mesmo. Para futuras análises a tela do vídeo de captura foi gravado permitindo a, posterior, contagem de 23 faces transitadas pela câmera, onde o algoritmo conseguiu detectar 16 delas, a qual 14 foram classificadas corretamente. (As faces foram borrada manualmente para preservar a identidade dos indivíduos)

Figura 5. Print de uma deteç̧ão realizada em alto fluxo de pessoas
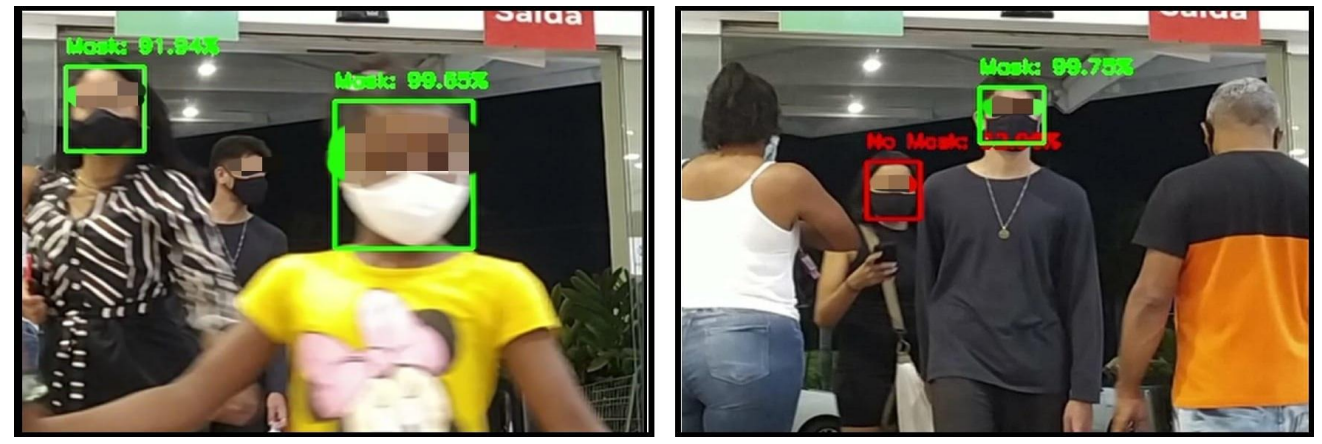

Fonte: Captura realizada pelo autor

superaram as expectativas em relação às métricas adotadas, dando continuidade ao trabalho será elaborado um modelo de armazenamento de dados, para captura de dados levantados pela correta classificação das faces.

\section{Conclusão}

Após ser testada em um ambiente de alto fluxo de pessoas, pode-se analisar, através da figura anterior, retirada do vídeo de detecção, que o algoritmo falha em detectar as faces quando a câmera está distante dos rostos, acarretando na não classificação das faces, também foi possível notar que a luz e ângulos, dos rostos, acabavam por interferir na classificação, pois quando o rosto era detectado nem sempre a classificação estava correta. Vale lembrar que a captura e estrutura montada para a mesma não foi a ideal, pois a ocasião não permitia, uma vez que se tratou de uma situação experimental a fim de ter parâmetros iniciais para um futuro novo teste mais estruturado, contudo pode-se observar que o algoritmo consegue sim lidar com múltiplas detecções, além de conseguir manter um fluxo de vídeo agradável e contínuo.

Por fim, avalia-se a ideia de implementar a detecção de áreas de alto fluxo como algo possível de se realizar com o algoritmo, entretanto para obter uma melhor implementação estudasse a melhoria do algoritmo para conseguir realizar, automaticamente, uma contagem de quantas classificações o mesmo conseguiu parametrizar e armazená-las em um banco de dados, a fim de ter esses dados disponíveis online, com acesso remoto.

Conclui-se que, o algoritmo proposto pode contribuir para subsidiar a tomada 
de decisões a partir dos dados e das estatísticas geradas pela solução, além ser utilizado para outros fins, uma vez que, é possível adaptar o método classificador do algoritmo, não limitando-se a somente classificar faces com e sem máscara.

\section{Referências}

Andrew G. Howard, Menglong Zhu, Bo Chen, Dmitry Kalenichenko, Weijun Wang, Tobias Weyand, Marco Andreetto, Hartwig Adam. MobileNets: Efficient Convolutional Neural Networks for Mobile Vision Applications. Cornell University. Disponível em: <https://arxiv.org/abs/1704.04861> Acesso em 05 maio 2021

Bertozzo, Bruno Henrique; BREZINSKI, Thiago. DESENVOLVIMENTO DE SKILL CLASSIFICADORA DE IMAGENS PARA AMAZON ALEXA. 2021. 75 f. TCC (Doutorado) - Curso de Sistemas de Informação, Departamento de Informática e Estatística, Universidade Federal de Santa Catarina, Florianópolis - Sc, 2021. Disponível em: https://repositorio.ufsc.br/bitstream/handle/123456789/228239/TCC\%20-\%20Dese nvolvimento $\% 20 \mathrm{de} \% 20$ skill $\% 20$ classificadora $\% 20 \mathrm{de} \% 20$ imagens $\% 20$ para $\% 20 \mathrm{Am}$ azon\%20Alexa.pdf?sequence=1\&isAllowed=y. Acesso em: 13 out. 2021.

BRASIL. UNIÃO. LEI No 14.019, DE 2 DE JULHO DE 2020. 2020. Órgão: Atos do Poder Legislativo. Disponível em: https://www.in.gov.br/en/web/dou/-/lei-n-14.019-de-2-de-julho-de-2020-264918074 . Acesso em: 20 jul. 2021.

DIDÁTICA TECH. O que é TensorFlow? Para que serve? 2020. Disponível em: https://didatica.tech/o-que-e-tensorflow-para-que-serve/. Acesso em: 25 jul. 2021.

Garcia, Leila Posenato. Uso de máscara facial para limitar a transmissão da COVID-19. Epidemiol. Serv. Saúde, Brasília, v.29, n.2, e2020222, 2020. Epub 22-Abr-2020.

Disponível

em: <https://doi.org/10.5123/S1679-49742020000200021.> Acesso em 24 julho 2021.

KERAS. About Keras. Disponível em: https://keras.io/about/. Acesso em: 25 jul. 2021.

MARIO FILHO,. As Métricas Mais Populares para Avaliar Modelos de Machine Learning. Disponível em: https://www.mariofilho.com/as-metricas-mais-populares-para-avaliar-modelos-de-mach ine-learning/. Acesso em: 17 out. 2021.

Resende, C. A. de P., \& Pereira, M. H. R. (2015). Visão Computacional Aplicada Em Reconhecimento Facial Na busca Por Pessoas Desaparecidas. E-Xacta, 8(2), 95-107. Disponível em: <http://dx.doi.org/10.18674/exacta.v8i2.1661> Acesso em 05 maio 2021.

VAN der Sande M, TEUNIS Peter, SABEL Rob. Professional and Home-Made FaceMasks Reduce Exposure to Respiratory Infections among the General Population. PLOS ONE. Disponível em: <https://doi.org/10.1371/journal.pone.0002618> Acesso em 05 maio 2021.

Zhao, W. et al. Face recognition: A literature survey. ACM Comput. Surv. 35, pp. 99-458, 4 Dez. 2003. 